ISSN electrónico: 2445-1355

DOI: https://doi.org/10.14201/fj2021617584

\title{
CESIÓN DE FÁRMACOS DESDE LIPOSOMAS EN DISTINTOS MEDIOS BIORRELEVANTES
}

\section{Drug Delivery from Liposomes in Different Biorelevant Media}

Azahara I. TORO; M. ${ }^{a}$ José de JESÚS VALLE

Departamento de Ciencias Farmacéuticas. Área de Farmacia y Tecnología Farmacéutica. Facultad de Farmacia. Campus Miguel de Unamuno. Licenciado Méndez Nieto, s/n. 37007 Salamanca

Correo-e: mariajosedj@usal.es

RESUMEN: Los liposomas constituyen sistemas vesiculares ideales para la liberación controlada y vectorización de fármacos a través de su administración pulmonar, debido a su similitud con las membranas celulares y su gran versatilidad.

El citrato de sildenafilo representa un óptimo modelo de fármaco para ser incorporado en estas vesículas lipídicas para el tratamiento de la hipertensión pulmonar.

El objetivo del trabajo fue caracterizar el comportamiento de los liposomas cargados con sildenafilo y evaluar la cesión del fármaco en distintos medios biorrelevantes.

Se elaboraron liposomas de dos tamaños ( 0.45 y 0.20 micras) mediante sonicación. Posteriormente se llevó a cabo el ensayo de liberación del fármaco en diferentes medios biorrelevantes (fluido plasmático simulado SBF, fluido pulmonar simulado SPF y PBS).

Según los resultados obtenidos se observó una velocidad de liberación del fármaco más sostenida y constante a lo largo del tiempo en el caso de los liposomas, independientemente del medio. En los medios PBS y SPF, se liberó una mayor cantidad de fármaco desde los liposomas con mayor tamaño $(0.45 \mu \mathrm{m})$, sin embargo, en el SBF las cinéticas de cesión de ambos 
tamaños de liposomas ( 0.45 y $0.20 \mu \mathrm{m})$ son muy similares, no encontrándose diferencias significativas en el tiempo o cantidad de fármaco liberada.

Palabras clave: liposomas; sildenafilo; medios biorrelevantes; hipertensión pulmonar.

ABSTRACT: Liposomes provide ideal vesicular systems for controlled release and vectorization of drugs through pulmonary administration, due to their similarity to cell membranes and their great versatility.

Sildenafil citrate represents an optimal model of drug to be incorporated into these lipid vesicles for the treatment of pulmonary hypertension.

The objective of the work was to characterize the behavior of liposomes loaded with sildenafil and evaluate the transfer of the drug in different bio-resistant media.

Liposomes of two sizes ( 0.45 and 0.20 microns) were developed by sonication. Subsequently, the drug release test was carried out in different biorelevant media (simulated plasma fluid SBF, simulated pulmonary fluid SPF and PBS).

According to the results obtained, a more sustained and consistent rate of release of the drug was observed over time in the case of liposomes, regardless of the medium. In PBS and SPF media, a greater amount of drug was released from the larger liposomes ( 0.45 microns), however, in the SBF the transfer kinetics of both liposome sizes $(0.45 \mu \mathrm{m}$ and $0.20 \mu \mathrm{m})$ are very similar, with no significant differences in the time or amount of drug released.

Keywords: liposomes; sildenafil; biorelevant media; pulmonary hypertension.

\section{INTRODUCCIÓN}

Los liposomas fueron descubiertos en 1960 por Alec D Bangham. Son estructuras microscópicas vesiculares concéntricas constituidas por membranas lipídicas dispuestas en bicapas y cuyo compartimento central es acuoso. Pueden ser unilaminares, con una única bicapa lipídica, o multilaminares, con más de una.

Son un sistema ideal para el transporte o reservorio de fármacos debido a sus propiedades. Morfológicamente son similares a las membranas celulares y pueden incorporar múltiples sustancias hidrofílicas e hidrofóbicas como fármacos, tóxicos, proteínas, enzimas. Actualmente son considerados unos prometedores sistemas de liberación controlada y de vectorización de fármacos muy investigados en 
diversos campos biomédicos (Bozzuto et al., 2015; Daraee et al., 2016; Javeri, Nellaiappan, 2018; De Jesús Valle, Sánchez, 2016).

El fármaco seleccionado es sildenafilo, un potente vasodilatador inhibidor de la fosfodiesterasa $\mathrm{V}$, por su creciente importancia en el tratamiento de la hipertensión pulmonar y otras patologías pulmonares como la displasia broncopulmonar o la hipertensión persistente en recién nacidos (Lavie et al., 2019; Villanueva et al., 2019; Van der Graaf et al., 2019).

La vía pulmonar ha sido estudiada para la administración no invasiva de fármacos con el fin de obtener efectos tanto locales como sistémicos, ya que presenta numerosas ventajas frente a la vía oral: una superficie amplia, favoreciendo la rápida absorción, una alta vascularización, menos efectos secundarios y menor efecto de primer paso (Paranjpe, Müller, 2014). El uso de sildenafilo por esta vía aumenta su vida media y además disminuye la incidencia de reacciones adversas (Shahin et al., 2019; Simonca, Tulloh, 2017).

Como vía de administración clásica, la vía parenteral, se considera útil su estudio para la administración sistémica de sildenafilo. Por lo general, el uso de esta vía se asocia a situaciones de urgencia en las que se necesita un efecto rápido, pero con el avance de la terapéutica y de la tecnología farmacéutica es posible su utilización también para la administración de sistemas de liberación controlada (Marques et al., 2011).

\section{2. Овјетivo}

El objetivo de este trabajo es caracterizar el comportamiento de liposomas unilaminares y evaluar la cesión de sildenafilo desde estas vesículas lipídicas, en distintos medios biorrelevantes, como el fluido pulmonar. Pues supone un paso importante para emplearlos en preparaciones inhalatorias destinadas al tratamiento de las patologías ya mencionadas.

\section{Materiales y métodos}

Citrato de sildenafilo como fármaco para ser incorporado a las vesículas lipídicas. Baño de ultrasonido ATU, incubador con agitación Labnet, pH-metro PEAK, centrífuga eppendorf 5418R, tubos pyrex 20.

\subsection{Preparación de liposomas}

Se realizan 3 lotes de liposomas de $0.20 \mu \mathrm{m}$ y otros 3 lotes de $0.45 \mu \mathrm{m}$ para el ensayo de cesión en cada medio biológico estudiado. El método empleado para su

Ediciones Universidad de Salamanca / @@ $\quad$ FarmaJournal, vol. 6, núm. 1 (2021), pp. 75-84 
preparación se caracteriza por la ausencia de utilización de disolventes orgánicos (De Jesús Valle, Sánchez, 2016).

Se mezclan los diferentes componentes, fosfatidilcolina y colesterol y mediante agitación se incorpora el fármaco, citrato de sildenafilo, en forma de solución acuosa, previamente disuelto en un tampón citrato, a un pH 3.2. Se agita para favorecer la suspensión de los lípidos y se añade un volumen total de $20 \mathrm{~mL}$ de citrato de sildenafilo.

Se preparan los liposomas por sonicación, introduciendo la suspensión de lípidos en un baño de ultrasonidos durante 30 minutos a $40^{\circ}$ y $50 \mathrm{~Hz}$. Se cambia la posición de la suspensión a los 15 minutos de iniciado el proceso para eliminar la posible variabilidad que pueda aparecer debido a la posición en la que se encuentre en el baño (De Jesús Valle, Sánchez, 2016; Sánchez et al., 2016).

Una vez finalizada la sonicación, para asegurar que se obtienen los liposomas del tamaño deseado, se somete la suspensión a extrusión. La extrusión es una técnica en la que la suspensión de liposomas se pasa a través de un filtro de tamaño de poro controlado repetidas veces (Ming Ong et al., 2016). Se realiza el método 10 veces con cada lote de liposomas, en este caso se elaboran liposomas de 0.45 $\mu \mathrm{m}$ y de $0.20 \mu \mathrm{m}$, como ya se ha comentado.

Tras la extrusión se deja reposar la suspensión 1 h a temperatura ambiente en un frasco topacio, protegido de la luz, donde se organizarán definitivamente los lípidos formando los liposomas y posteriormente deberán pasar mínimo otra hora refrigerados.

\subsection{Encapsulación de sildenafilo}

Tras finalizar ambos períodos de reposo en los que ya se han formado finalmente los liposomas, el siguiente paso es conseguir la encapsulación del sildenafilo dentro de las vesículas, para lo que se establece un gradiente de $\mathrm{pH}$ entre el interior del liposoma y el medio exterior. Mientras que el interior se encuentra a un $\mathrm{pH}$ de 3.2, en el medio externo se instaura un $\mathrm{pH}$ de 7 con ayuda de $\mathrm{NaOH} 1 \mathrm{M}$.

Se pasa la suspensión de liposomas a un baño de incubación en el que la temperatura se fija a $25^{\circ} \mathrm{C}$ y la agitación a $40 \mathrm{rpm}$ durante 20 horas.

El sildenafilo a pH 7 se encuentra en forma molecular, es decir, no ionizado, por lo que tiene capacidad para atravesar las membranas lipídicas de los liposomas. En el interior de las vesículas el fármaco se ioniza debido a la existencia del pH ácido (3.2), quedando de esta forma encapsulado. Dicho proceso tiene lugar a lo largo de estas 20 horas. Una vez finalizado este período de tiempo, los liposomas están preparados para poder realizar el ensayo de cesión, hasta el cual se pueden conservar refrigerados (De Jesús Valle et al., 2018). 


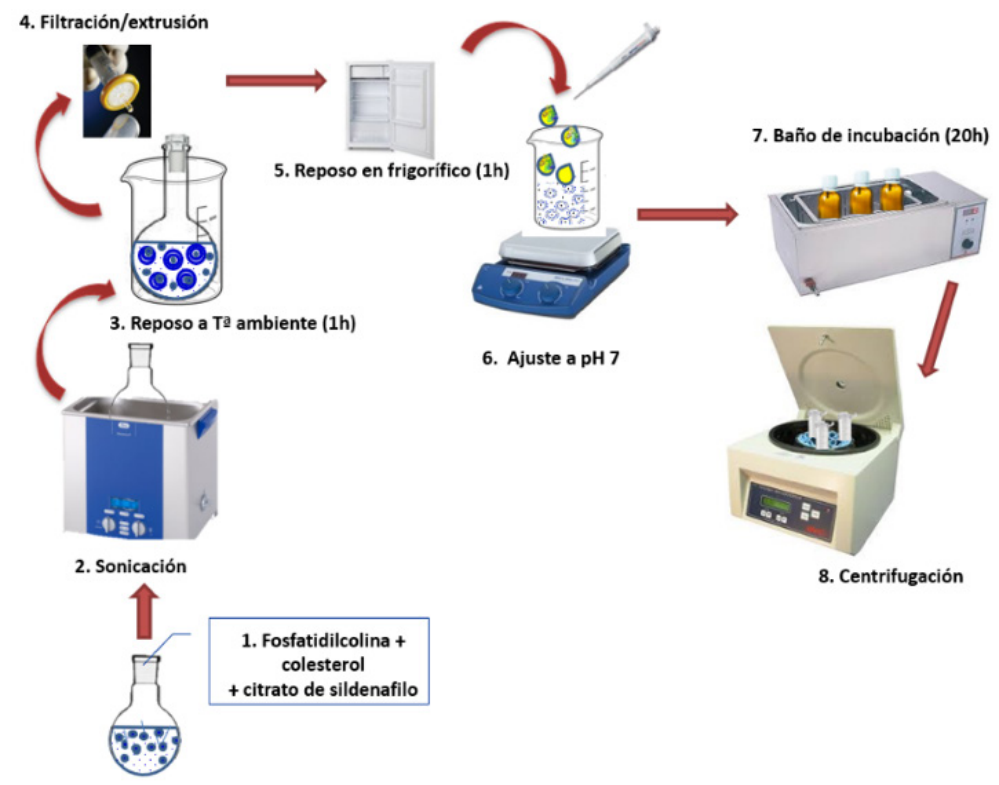

Figura 1. Preparación de liposomas.

\subsection{Ensayo de cesión}

Este ensayo se realiza en cada medio biorrelevante con 3 lotes de liposomas de 0.45 micras y 3 lotes de liposomas de 0.20 micras, junto con las referencias correspondientes, en las cuales el fármaco no está encapsulado en liposomas.

Se centrifugan $2 \mathrm{ml}$ de cada lote durante 45 minutos en eppendorfs y se desecha el sobrenadante para eliminar todo el sildenafilo que haya quedado en el medio, sin encapsular, para que no afecte a la cesión. Se reconstituye con el medio correspondiente en cada caso y se introduce $1 \mathrm{~mL}$ de cada lote en la respectiva membrana de diálisis formando un saco cerrado por ambos extremos que se introduce en tubos pirex de 20. Las referencias incluyen en la membrana de diálisis sildenafilo sin encapsular, el resto se elabora de la misma manera.

Una vez estén todos los tubos con las membranas preparadas, se introducen $10 \mathrm{~mL}$ del medio a ensayar; rápidamente, se incorporan al baño con agitación a la temperatura fisiológica de $37^{\circ} \mathrm{C}$ y muestreamos a determinados tiempos previamente establecidos: $15 \mathrm{~min}, 30 \mathrm{~min}, 45 \mathrm{~min}, 1 \mathrm{~h}, 1.5 \mathrm{~h}, 2 \mathrm{~h}, 3 \mathrm{~h}, 4 \mathrm{~h}, 6 \mathrm{~h}, 10$ h, 24 h, 30 h y 48 h. 
Se cogen $100 \mu \mathrm{L}$ en cada muestra que se introducen en viales para determinar la cantidad de sildenafilo mediante cromatografía líquida de alta eficacia (HPLC).

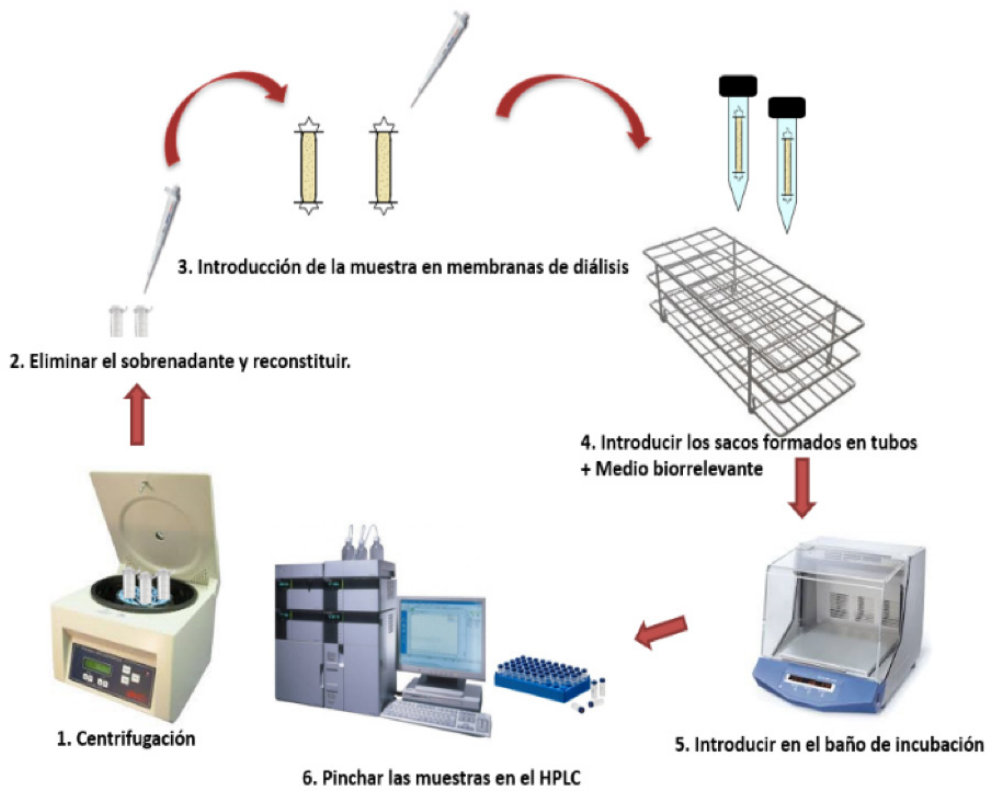

Figura 2. Ensayo de cesión.

\subsection{Medios biorrelevantes}

Se utilizó como medio de referencia PBS (phosphate buffered saline), que es una solución cuya osmolaridad y concentración iónica es similar al líquido extracelular, frente al cual se comparó la liberación de sildenafilo en fluido plasmático simulado (SBF) o en fluido pulmonar simulado (SPF) (Kumar et al., 2017).

\section{Resultados y discusión}

En algunos medios observamos, al inicio de las curvas de cesión, una ligera variabilidad, sobre todo en las referencias, que pueden deberse a tiempos demasiado cortos en el muestreo en las etapas iniciales. Igualmente, un aparente descenso en la concentración de sildenafilo en las últimas muestras.

Como cabe esperar, en las referencias la velocidad de cesión es muy rápida en las primeras muestras y desciende en las posteriores. Mientras que, en el caso de los liposomas, la velocidad es más sostenida y constante a lo largo del tiempo, ya que se trata de un sistema de liberación controlada.

Ediciones Universidad de Salamanca / @®@ FarmaJournal, vol. 6, núm. 1 (2021), pp. 75-84 
En los medios PBS y SPF, se libera una mayor cantidad de fármaco desde los liposomas con mayor tamaño $(0.45 \mu \mathrm{m})$, sin embargo, en el medio SBF las cinéticas de cesión de ambos tamaños de liposomas $(0.45$ y $0.20 \mu \mathrm{m})$ son muy similares, no encontrándose diferencias significativas en el tiempo o cantidad de fármaco liberada.

La similitud en el medio SBF entre los liposomas de 0.45 y 0.20 micras es muy interesante ya que por vía parenteral nos interesan los liposomas de menor tamaño para cumplir con los requisitos de tamaño y esterilidad que la caracterizan.

En la Figura 3 pueden observarse ligeras diferencias entre la cesión del fármaco desde los liposomas de 0.45 micras y los de 0.20 micras, siendo esta diferencia mayor respecto a las referencias en los medios PBS y SBF.
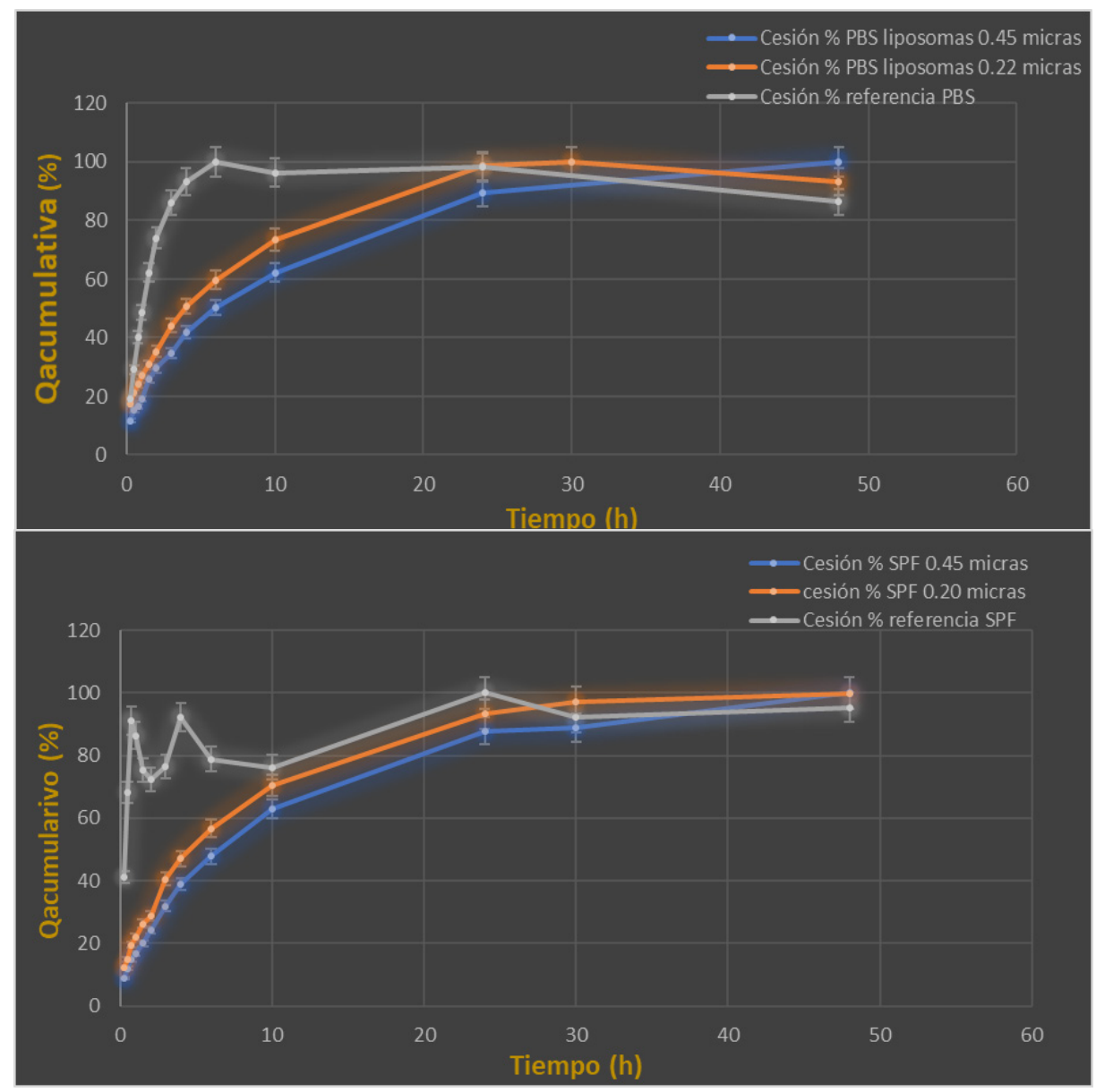

Ediciones Universidad de Salamanca /

FarmaJournal, vol. 6, núm. 1 (2021), pp. 75-84 
AZAHARA I. TORO; M. ${ }^{a}$ JOSÉ DE JESÚS VALLE

CESIÓN DE FÁRMACOS DESDE LIPOSOMAS EN DISTINTOS MEDIOS BIORRELEVANTES
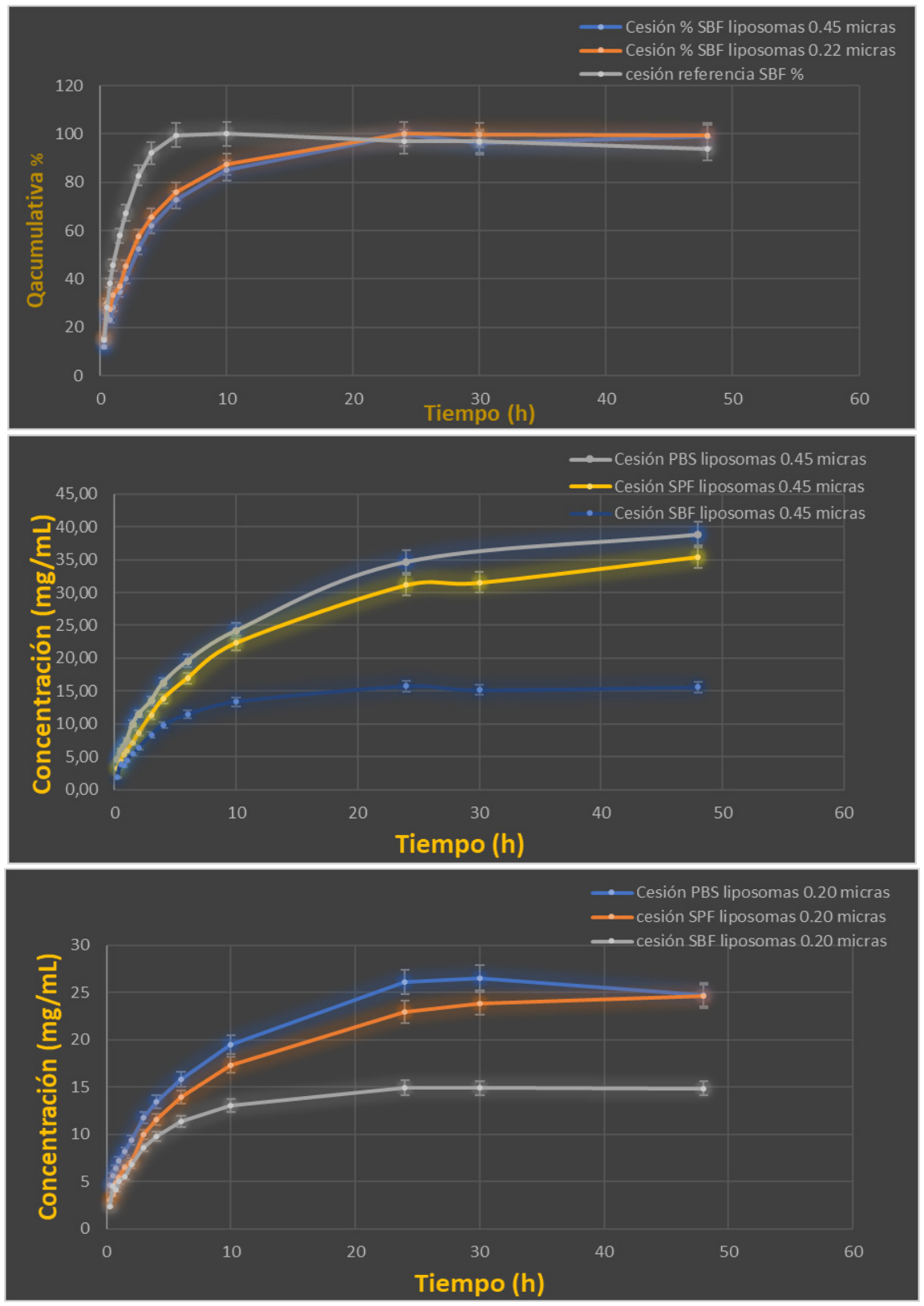

Figura 3. Cesión de sildenafilo en los medios biorrelevantes.

Ediciones Universidad de Salamanca / @®@

FarmaJournal, vol. 6, núm. 1 (2021), pp. 75-84 
De manera general se observa una mayor cesión de sildenafilo en el fluido pulmonar simulado que en el plasma simulado, siendo menos marcada la diferencia en los liposomas de menor tamaño.

\section{Conclusiones}

Este trabajo constituye una etapa inicial en el estudio y evaluación de la cesión de sildenafilo desde vesículas de diferente tamaño y varios medios biorrelevantes. En la actualidad continúan realizándose ensayos, con un número mayor de lotes, para poder determinar y evaluar la influencia de estos parámetros en la cesión del fármaco desde los liposomas.

Pero teniendo en cuenta los resultados obtenidos en el presente trabajo, se puede deducir que los liposomas son formas farmacéuticas muy interesantes desde el punto de vista de una liberación prolongada, existiendo una cesión del fármaco durante las 48 horas de muestreo, pudiendo suponer una simplificación en las pautas de dosificación.

Además de conseguir una velocidad constante y controlada en la liberación del fármaco, pudiendo mantener las concentraciones de sildenafilo en su margen terapéutico, lo cual es muy útil para aquellos fármacos que presentan un rango estrecho.

\section{Bibliografía}

Bozzuto G, Molinari A. Liposomes as nanomedical devices. International Journal of Nanomedicine. 2015; (10):975-999.

Daraee H, Etemadi A, Kouhi M, Alimirzalu S, Akbarzadeh A. Application of liposomes in medicine and drug delivery. Artificial Cells, Nanomedicine and Biotechnology. 2016; 4(4):381-391.

De Jesús MJ, Gil P, Prata M, Araujo A RTS, Sánchez A. Sildenafil Citrate Liposomes for Pulmonary Delivery by Ultrasonic Nebulization. Applied sciences. 2018; (8).

De Jesús Valle MJ, Sánchez Navarro A. Liposomes prepared in absence of organic solvents: sonication versus lipid film hydration method. Current pharmaceutical análisis. 2015; (11):86-91.

E. Villanueva DL, Divine Agustin R, Jasper Llanes E. Pre-operative sildenafil for patients with pulmonary hypertension undergoing mitral valve surgery: a systematic review and meta-analysis. Elmer press. 2019; (10):369-377.

Javeri I, Nellaiappan K. Methods for the preparation of liposomes. US. 10, 143, 652 B2. 2018. Kumar A, Terakosolphan W, Hassoun M, Vandera KK, Novicky A, Harvey R et al. A Biocompatible Synthetic Lung Fluid Based on Human Respiratory Tract Lining Fluid Composition. Pharmaceutical Research. 2017; (34):2454-2465. 
Lavie-Nevo K, C. Harris K, Y. Ting J. Use of sildenafil in an infant with persistent pulmonary hypertension secondary to lung and renal hipoplasia- a case report. BMC pedriatics. 2019; (19):416.

Marques MRC, Loebenberg, R. Almukainzi, M. Simulated biological fluids with possible application in dissolution testing. Dissolution technologies. 2011; (18):15-28.

Ming Ong SG, Chitneni M, Seng Lee K, Chiau Ming L, Hay Yuen K. Evaluation of Extrusion Technique for Nanosizing Liposomes. Pharmaceutics. 2016; (8).

Paranjpe M, Müller-Goymann CC. Nanoparticle-Mediated Pulmonary Drug Delivery: A Review. International Journal of Molecular Sciences. 2014; (15):5852-5873.

Sánchez MJ, Sánchez A, De Jesús MJ. Caracterización y separación de liposomas por microencapsulación. FarmaJournal. 2016; (1):93-100.

Shahin HI, Vinjamuri BP, Mahmoud AA, Shamma RN, Mansour SM, Ammar HO et al. Design and Evaluation of Novel Inhalable Sildenafil Citrate Spray-Dried Microparticles for Pulmonary Arterial Hypertension. Journal of Controlled Release. 2019; (302):126-139.

Simonca L, Tulloh T. Sildenafil in Infants and Children. Children. 2017; (4).

Van der Graaf M, Arindah Rojer L, Arnold Helbing W, Marcel Reiss IK, Gregory Etnel JR, Bartelds B. Sildenafil for bronchopulmonary dysplasia and pulmonary hypertension: a meta-analysis. Pulmonary circulation. 2019; (9):1-8. 